\title{
A importância da luz na ocupação de árvores por lianas
}

\author{
The importance of light for liana occupation on trees
}

Julia Caram Sfair ${ }^{1,3}$,Bruno Roberto Ribeiro ${ }^{2}$ Erlon Pessoni Pimenta ${ }^{2}$, Tamires Gonçalves ${ }^{2}$ \& Flavio Nunes Ramos ${ }^{2}$

\begin{abstract}
Resumo
Lianas interagem de forma negativa com as árvores, uma vez que aumentam suas taxas de mortalidade. Seria esperado, portanto, que árvores apresentassem possíveis caracteres que as defenderiam contra lianas. O objetivo desse estudo foi testar se os seguintes caracteres das árvores limitam sua infestação por lianas: 1) baixa altura; 2) tronco com ramificações altas (fuste alto); 3) casca lisa; 4) casca esfoliante, e 5) menor índice de iluminação da copa. O estudo foi realizado em um fragmento de Floresta Estacional Semidecídua no município de Alfenas (MG) no qual foi amostrado em 0,1 ha, todas as árvores com diâmetro na altura do peito maior do que $3 \mathrm{~cm}$. Para cada árvore registrou-se os caracteres citados anteriormente, bem como foi estimada a porcentagem de cobertura de lianas na copa. Encontrou-se que quanto maior a altura e o índice de iluminação da copa, maior a sua ocupação por lianas. Essas árvores, apesar de atingirem o dossel da floresta e, portanto, adquirirem mais luz para crescimento e reprodução, estão também mais sujeitas à ocupação por lianas. Apesar disso, supõe-se que as árvores não investem em estruturas que poderiam diminuir a ocupação por lianas, provavelmente porque a competição com outras plantas da vizinhança pode ser também intensa. Palavras-chave: competição, comunidade vegetal, Floresta Estacional Semidecídua, trepadeira.
\end{abstract}

\begin{abstract}
Lianas (woody climbers) interact negatively with trees, increasing their mortality rates. We expect, therefore, that trees have developed supposed traits that would defend them against lianas. The aim of this paper was test if these tree traits limit their liana occupation: 1) smaller height; 2) taller free bole height; 3) smooth bark; 4) exfoliating bark, and 5) low crown illumination. This study was carried out in a fragment of a seasonal semideciduous forest in Alfenas (Minas Gerais; Southeastern Brasil). We sampled in 0.1 ha all trees with diameter at breast height larger than $3 \mathrm{~cm}$. For each tree we noted the traits previously cited, as well as we estimated the percentage of liana occupation on the tree crowns. We found that, for taller trees and trees with higher crown illumination, the probability of a tree to carry lianas was higher. These trees, in spite of reaching the forest canopy, and, therefore, acquire more light for reproduction and growth, they are also more prone to liana occupation. In spite of that, we suppose that the trees do not invest on traits that could decrease the liana occupation, probably because the competition with neighbor's plants may also be intense.
\end{abstract}

Key words: competition, plant community, Semideciduous Forest, climber.

\section{Introdução}

Lianas ou trepadeiras lenhosas, de acordo com terminologia de Gerwing et al. (2006), interagem de maneira negativa com as árvores, diminuindo sua produção de frutos (Kainer et al. 2006; Fonseca et al. 2009) e crescimento (Campanello et al. 2007). Consequentemente, algumas espécies de forófitos (plantas hospedeiras) possuem maior taxa de mortalidade do que árvores sem lianas (Grogan \& Landis 2009). Esses efeitos negativos sobre o forófito poderiam explicar a presença de supostos caracteres defensivos das árvores contra lianas (Hegarty 1991).

Hegarty (1991) cita diversas estruturas que as árvores possuem que poderiam diminuir a sua ocupação por lianas. Dentre essas estruturas há troncos com casca esfoliante ou lisa (Hegarty

\footnotetext{
${ }^{1}$ Universidade Federal de Pernambuco, Departamento de Botânica, Av. Prof. Moraes Rego 1235, Cidade Universitária, 50670-901, Recife, PE, Brasil.

${ }^{2}$ Universidade Federal de Alfenas - UNIFAL-MG, Inst. Ciências da Natureza, Lab. Ecologia de Fragmentos Florestais - ECOFRAG, R. Gabriel Monteiro da Silva 700, 37130-000, Alfenas, MG, Brasil.

${ }^{3}$ Autor para correspondência: juliacaram@gmail.com
} 
1991). A casca esfoliante está relacionada à perda da estabilidade no local de aderência da liana (Hegarty 1991), enquanto que as com cascas lisas possuem menos pontos de aderência para lianas, comparadas às de cascas rugosas; estas últimas conferem maior área para lianas se agarrarem (Talley et al. 1996).

A arquitetura e o tamanho da árvore também podem dificultar a ocupação por lianas (Hegarty 1991). Por exemplo, sabe-se que árvores maiores tendem a ter mais lianas (Clark \& Clark 1990; Ding \& Zang 2009; Jiménez-Castillo \& Lusk 2009; Homeier et al. 2010). Uma explicação é que essas árvores mais altas atingem o dossel da floresta e, portanto, recebem mais luz na copa (Malizia \& Grau 2006). Dessa maneira, espera-se que copas mais iluminadas também possuam mais lianas, como observado por Malizia \& Grau (2006). Outra variável relacionada à ocupação por lianas é a altura do fuste ou o tamanho relativo do caule sem ramificações (Campbell \& Newbery 1993; Muthuramkumar \& Parthasarathy 2001; Reddy \& Parthasarathy 2006). Quanto menos ramificações ou galhos baixos uma árvore possui, menor o número de locais em que uma liana pode se fixar. Além disso, com o crescimento da árvore, há a queda de galhos mais baixos, que podem conter lianas (Campbell \& Newbery 1993). Dessa maneira, árvores com muitos galhos em alturas relativamente baixas teriam mais lianas do que árvores com maior porção do tronco sem galhos (Reddy \& Parthasarathy 2006).

Tendo em vista os efeitos negativos que as lianas exercem sobre as árvores, algumas das estruturas que as árvores possuem limitariam a sua colonização por trepadeiras lenhosas (Hegarty 1991). Dessa maneira, o objetivo do presente estudo foi responder se os seguintes caracteres das árvores estão relacionados à sua ocupação por lianas: altura total; altura do fuste em relação à altura total; tipo de casca do tronco, e índice de iluminação da copa.

Esperamos que árvores baixas, com ramificações altas em relação à altura total, com casca lisa e com baixo índice de iluminação da copa sejam menos ocupada por lianas (Hegarty 1991), uma vez que seriam caracteres das árvores que limitariam sua ocupação pelas mesmas.

\section{Material e Métodos}

Realizamos o estudo em um fragmento de Floresta Estacional Semidecídua localizado no município de Alfenas (Minas Gerais). A região possui temperatura média anual em torno de $23^{\circ} \mathrm{C}$ e precipitação média anual de $1.513 \mathrm{~mm}$ (Costa 1998). O fragmento $\left(21^{\circ} 33^{\prime} 44,68^{\prime \prime} \mathrm{S}\right.$ e $45^{\circ} 56^{\prime} 12,80^{\prime} \mathrm{O}$; $870 \mathrm{~m}$ de altitude) possui área total de 49,68 ha e está imerso em uma matriz de cultura de café, possuindo muitas clareiras, e, consequentemente, lianas e bambus. Seu histórico de perturbação não é conhecido.

Distribuímos cinco parcelas de $10 \times 20 \mathrm{~m}$, totalizando $1.000 \mathrm{~m}^{2}$ de área amostral $(0,1 \mathrm{ha})$. As parcelas foram colocadas distantes pelo menos $200 \mathrm{~m}$ entre si, de maneira a amostrarmos porções independentes estatisticamente da floresta (Legendre \& Fortin 1989). As parcelas foram alocadas em pontos de floresta menos perturbados no interior do fragmento, de forma a evitar a amostragem em clareiras. Clareiras podem atrapalhar a coleta e as análises de dados, uma vez que possuem grande quantidade de bambus e de lianas, bem como uma baixa densidade de árvores. Em cada parcela amostramos todas as árvores com diâmetro na altura do peito maior ou igual a $3,0 \mathrm{~cm}$, uma vez que o dossel da floresta amostrada é bastante baixo (altura máxima das árvores é de $15 \mathrm{~m}$; Fig. 1). Seguindo o protocolo proposto por Gerwing et al. (2006) e Schnitzer et al. (2008), amostramos todas as lianas com diâmetro na altura do peito maior ou igual a $1,0 \mathrm{~cm}$. Observamos também qual porção da árvore a liana ocupava: tronco, copa ou ambos. As árvores e as lianas não foram identificadas até a categoria taxonômica de espécie; tampouco registramos o método de escalada de lianas.

Registramos os seguintes caracteres das árvores que poderiam influenciar sua ocupação por lianas: altura total, altura do fuste (primeira ramificação da árvore), casca do tronco lisa ou rugosa, casca do tronco esfoliante ou persistente e o índice de iluminação da copa (IIC). A altura total e a altura do fuste foram estimadas visualmente, utilizando como parâmetro, o tamanho da tesoura de alta poda, que possuía $6 \mathrm{~m}$ de altura. Consideramos a razão entre a altura do fuste e a altura total como sendo a proporção do tronco livre de galhos. Quanto maior a proporção (mais próxima de 1), maior a porção do caule sem galhos e, portanto, menor a probabilidade da árvore ter lianas. A categorização em casca lisa ou rugosa e em casca esfoliante ou persistente foram adaptadas de Torres et al. (1994).

O IIC é uma estimativa visual e está relacionado à quantidade e localização da luz na copa da árvore. A vantagem da sua utilização é a possibilidade de ser aplicado pelo pesquisador visualmente e no chão da floresta (Clark \& 


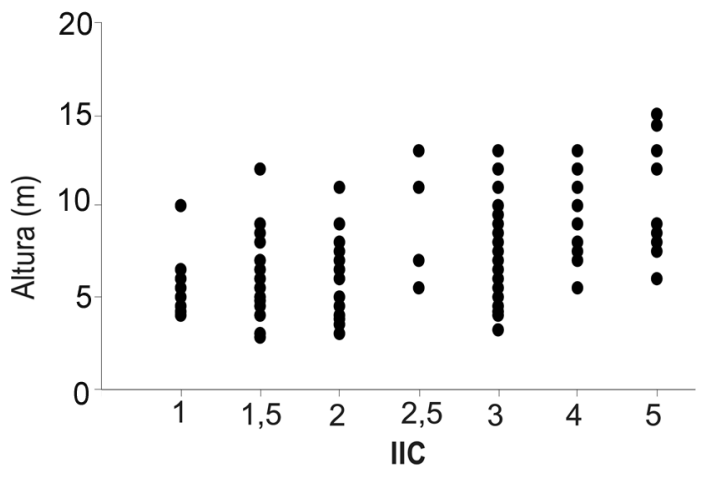

Figura 1 - Distribuição das alturas das árvores de acordo com o valor de índice de iluminação da copa(IIC). Quanto maior o IIC, maior a exposição da copa à luz.

Figure 1 - Distribution of tree height according to the canopy illumination index (CII). Higher CII indicates more exposition of the canopy by light.

Clark 1992), bem como para árvores que perdem totalmente as folhas, desde que a área da copa seja possível de ser estimada. Utilizamos o método de Dawkins \& Field (1978), adaptado por Clark \& Clark (1992), em que a copa da árvore pode receber luz de diferentes modos: 1 - sem luz direta vertical ou lateralmente; 1,5 - pouca luz lateral; 2 - média luz lateral; 2,5 - muita luz lateral; $3-10-90 \%$ da copa exposta à luz vertical, mas menos de $10 \%$ da copa exposta à luz lateral; 4 - mais de $90 \%$ da copa exposta à luz vertical e até $90 \%$ de luz lateral; 5 - copa completamente exposta, lateral e verticalmente (Clark \& Clark 1992). Dessa maneira, há um gradiente de exposição de copa, que vai de copas totalmente sombreadas (IIC $=1$ ) até aquelas totalmente expostas (IIC $=5$ ).

Os testes estatísticos referentes à casca rugosa e à casca esfoliante levaram em consideração a presença ou ausência de lianas no tronco no momento da amostragem, enquanto que os testes referentes à altura da árvore, à proporção do tronco livre de galhos e ao IIC levaram em consideração a estimativa de cobertura de lianas na copa das árvores no momento da amostragem. Estimamos essa cobertura seguindo as seguintes classes: 0 $0 \% ; 1$ - 1 a 25\%; 2 - 26 a 50\%; 3 - 51 a $75 \% ; 4$ - 76 a $100 \%$ da copa das árvores coberta por lianas (classe de cobertura). Utilizamos o teste de qui-quadrado para verificar a diferença no número de árvores entre essas classes.

Aplicamos análise de variância (ANOVA) para verificar se árvores mais altas possuem mais lianas. Optamos pela ANOVA porque os dados possuíam distribuição normal e eram homocedásticos, ou seja, possuíam variâncias homogêneas entre grupos (Zar 2010). Nestes testes, comparamos as alturas das árvores em diferentes categorias correspondentes à porcentagem de cobertura de lianas. Empregamos o teste de Tukey HSD a posteriori para verificar as diferenças significativas entre as médias dos pares de categorias. Para verificar se havia diferença na classe de cobertura de lianas em árvore com diferentes proporções do tronco livre de galhos, utilizamos o teste de Kruskal-Wallis. Esse teste não-paramétrico foi empregado como alternativa à ANOVA, pois estes dados não possuíam distribuição normal e as variâncias mantiveramse heterogêneas mesmo após transformações matemáticas para normalizar os dados (Zar 2010).

Para verificar se árvores com casca lisa possuem menos lianas em relação às árvores com casca rugosa, utilizamos o teste de qui-quadrado, que também empregamos para verificar a diferença no número de árvores que possuíam casca esfoliante e que possuíam casca persistente em relação ao número de lianas no caule. Para os dois testes consideramos apenas troncos que possuíam ou não lianas.

Utilizamos a correlação não-paramétrica de Kendall para verificar se há um aumento na classe de cobertura de lianas na árvore conforme o aumento no valor de IIC. A correlação de Kendall é indicada quando o número de observações é pequeno (Zar 2010). Empregamos esse mesmo teste estatístico para averiguar se árvores com IIC maior são mais altas. Realizamos todos os testes estatísticos no ambiente de programação $\mathrm{R}$ (R Development Core Team 2011) e se basearam nas recomendações presentes em Zar (2010). Realizamos as análises de Kendall por meio do pacote "Kendall" (McLeod 2011).

\section{Resultados}

Encontramos 136 indivíduos de árvores e 215 indivíduos de lianas. A altura média das árvores foi de 7,18 $\mathrm{m}( \pm 2,68 \mathrm{~m})$, sendo que a maior árvore possuía altura de $15 \mathrm{~m}$ (Fig. 1) e altura do fuste de $12 \mathrm{~m}$ e a menor, $2,8 \mathrm{~m}$ de altura e $0,4 \mathrm{~m}$ de fuste. A porcentagem de cobertura de lianas na copa da árvore variou $\left(\chi^{2}=14,81, p=0,005\right)$, sendo que a maioria das árvores não carregou lianas ou possuía de 1 a $25 \%$ ou de 51 a $75 \%$ de lianas na copa (Fig. 2).

As árvores com maior cobertura de lianas em suas copas (classe 4) apresentaram as maiores 


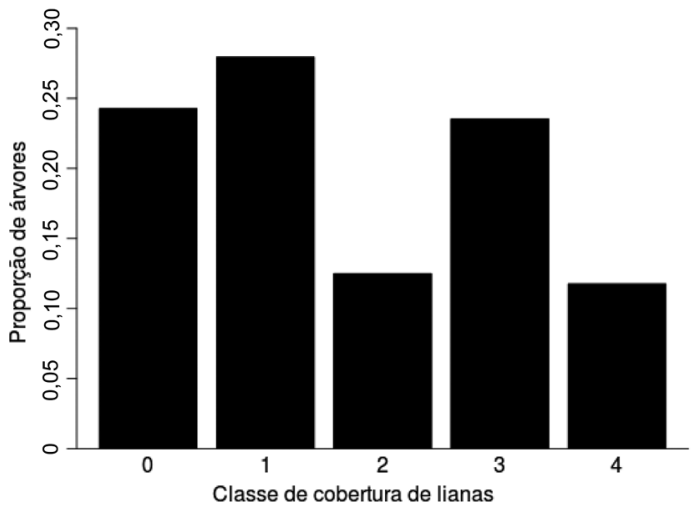

Figura 2 - Proporção do número de árvores de acordo com a classe de porcentagem de cobertura de lianas (0 - 0\% ; 1 - 1 a 25\%; 2 - 26 a 50\%; 3 - 51 a 75\%; 4 - 76 a $100 \%$ da copa das árvores coberta por lianas).

Figure 2 - Proportion of the number of trees according to the class of lianas coverage percentage $(0-0 \% ; 1-1$ to $25 \%$; 2 - 26 to $50 \% ; 3-51$ to $75 \%$; $4-76$ to $100 \%$ of the tree canopy covered by lianas).

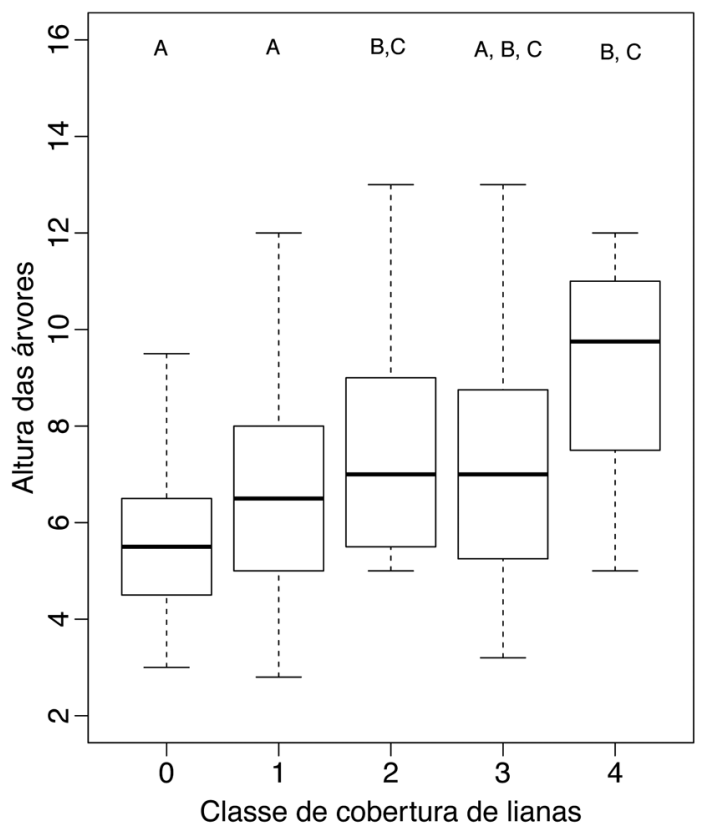

Figura 3 - Distribuição das alturas das árvores de acordo com a classe de porcentagem de cobertura de lianas na copa $(0-0 \% ; 1-1$ a $25 \% ; 2$ - 26 a $50 \% ; 3$ - 51 a $75 \% ; 4$ - 76 a 100\% da copa das árvores coberta por lianas). As letras indicam as diferenças nas classes de acordo com teste de Tukey.

Figure 3 - Distribution of tree height according to the class of lianas coverage percentage $(0-0 \% ; 1-1$ to $25 \% ; 2-26$ to $50 \% ; 3-51$ to $75 \% ; 4-76$ to $100 \%$ of the tree canopy covered by lianas). The letters indicate the differences between classes according to Tukey test. alturas e as árvores sem liana (classe 0), as menores $\left(\mathrm{F}_{4,131}=5,56, \mathrm{p}<0,001\right.$; Fig. 3). Árvores maiores possuem copas mais iluminadas $(\tau=0,43, \mathrm{p}<$ 0,001; Fig. 1) e, quanto maior sua iluminação (maior IIC), maior a porcentagem de cobertura de lianas $(\tau=0,34, \mathrm{p}<0,001)$.

Não houve diferenças significativas entre: (i) as classes de cobertura da copa por lianas com relação à altura do tronco livre de galhos $\left(\mathrm{KS}_{4}=4,20, \mathrm{p}=0,38\right.$; Fig. 4); (ii) no número de lianas em árvores com casca lisa e árvores com casca rugosa $\left(\chi_{4}^{2}=3,77, \mathrm{p}=0,44\right)$; e (iii) entre árvores com casca persistente e com casca esfoliante $\left(\chi_{4}^{2}=3,80, \mathrm{p}=0,43\right)$.

\section{Discussão}

Nesse estudo mostramos que a altura e a iluminação da copa de uma árvore estão relacionadas à ocupação por lianas, de maneira que, quanto maior a iluminação da copa e a altura da árvore, maior a sua probabilidade de ser colonizada por lianas. Há três explicações possíveis para a relação positiva entre altura e número de lianas: a. essas árvores

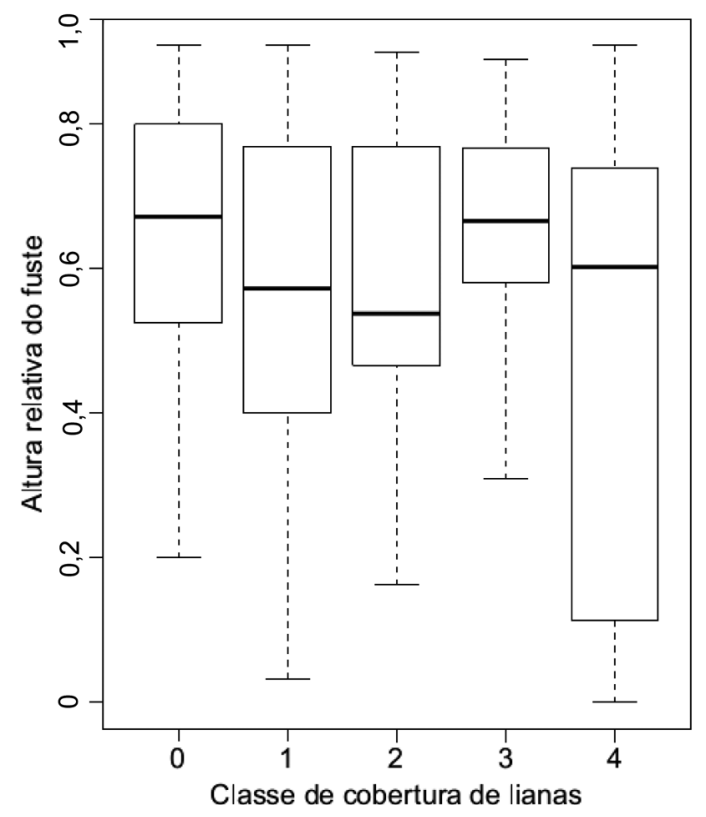

Figura 4 - Distribuição da altura relativa do fuste (altura do tronco livre de galhos) em relação à altura total da árvore de acordo com a porcentagem de cobertura de lianas (0 - 0\%; 1 - 1 a 25\%; 2 - 26 a 50\%; 3 - 51 a 75\%; 4 - 76 a $100 \%$ da copa das árvores coberta por lianas). Figure 4 - Distribution of free bole height in relation to the total height of the tree according to the percentage of tree covered by lianas $(0-0 \% ; 1-1$ to $25 \% ; 2-26$ to $50 \% ; 3-51$ to $75 \% ; 4-76$ to $100 \%$ of the tree canopy covered by lianas). 
seriam supostamente mais velhas e, portanto, forneceriam mais tempo para a colonização de lianas (Pérez-Salicrup et al. 2001; Malizia \& Grau 2006; Campanello et al. 2007); b. uma vez que uma liana atinge a copa da árvore, outras lianas seriam favorecidas por esse atalho, em um processo que poderia ser considerado "facilitação" (Pinard \& Putz 1994),e c. essas árvores mais altas atingem o dossel da floresta e, portanto, receberiam mais luz na copa (Malizia \& Grau 2006). A primeira explicação provavelmente não é satisfatória, pois árvores menores podem ser tão ou até mais velhas que as árvores altas, o que as tornariam também muito favorecidas à ocupação por lianas (Balfour \& Bond 1993; Chittibabu \& Parthasarathy 2001; Carsten et al. 2002). Essas árvores pequenas podem ainda servir de atalhos para as lianas ocuparem as árvores maiores e também poderiam ser tão ou mais infestadas por lianas quanto árvores maiores. A segunda explicação não pôde ser comprovada com os dados coletados nesse estudo, pois a facilitação poderia apenas ser comprovada para lianas volúveis e escandentes: lianas com gavinhas perdem essas estruturas à medida que sobem ao dossel da floresta. Acreditamos que para comprovar a facilitação, seria necessário acompanhar o crescimento da liana e a perda de suas gavinhas ao longo do tempo.

Apenas a terceira explicação relaciona-se aos resultados encontrados nesse estudo, uma vez que árvores mais altas tendem a ter copas mais iluminadas e, consequentemente, mais lianas. Dessa maneira, acreditamos que há uma correlação entre o fato da árvore ser alta, atingir o dossel da floresta, receber mais luz e ter maior porcentagem da copa ocupada por lianas.

As plantas são limitadas por luz e sua disponibilidade é fundamental para explicar a diversidade taxonômica e funcional entre as plantas em uma floresta tropical (Ghazoul \& Sheil 2010). Uma maior iluminação permite que as plantas adquiram mais energia para crescer, autorrepararem em caso de dano e se reproduzirem (Ghazoul \& Sheil 2010). Dessa maneira, árvores que atingem o dossel da floresta teriam a possibilidade de adquirir mais luz e, consequentemente, possuiriam vantagens competitivas em relação a árvores que recebem menos luz. Entretanto essas árvores que atingem o dossel da floresta estão mais propensas a serem ocupadas por lianas, tendo seu vigor adaptativo reduzido devido à diminuição da sua taxa de crescimento (Campanello et al. 2007) e de fecundidade (Kainer et al. 2006; Fonseca et al.
2009). Há, portanto, uma compensação (trade-off) para a árvore ao atingir o dossel da floresta, pois ao mesmo tempo em que ela pode adquirir mais luz, também está mais propensa à ocupação por lianas, que possuem uma relação negativa com as árvores (Kainer et al. 2006; Campanello et al. 2007; Fonseca et al. 2009; Grogan \& Landis 2009).

A ocorrência de caracteres que inibem a colonização por lianas poderia, portanto, ajudar a diminuir a probabilidade de uma árvore ser ocupada por lianas. Entretanto, os caracteres das árvores considerados neste estudo (casca esfoliante, casca lisa e maior porção do tronco livre de galhos) pareceram não ser suficientes para evitar a ocupação por lianas. A interação negativa entre forófito e liana pode não ser tão intensa. Há um consenso entre pesquisadores que a interação entre árvores e lianas seria do tipo competição (por exemplo, Schnitzer et al. 2005 e Chen et al. 2008). Muitos dos experimentos sobre competição entre árvores e lianas desconsideram a interação entre outras plantas da vizinhança, utilizando experimentos em casa de vegetação (como o estudo de Chen et al. 2008) ou em campo, mas de forma controlada, ou seja, isolando variáveis. Por exemplo, Schnitzer et al. (2005) plantaram indivíduos jovens de árvores na floresta e esperaram que lianas ocupassem os pequenos forófitos para ver o efeito da competição entre esses hábitos de vida acima e abaixo do solo. Entretanto, essas árvores jovens plantadas estavam distantes pelo menos $3 \mathrm{~m}$ de outras árvores (Schnitzer et al. 2005). Em um ambiente não controlado na floresta, essa árvore competiria por nutrientes, água e luz não somente com a liana, mas com outras plantas próximas a ela. Dessa maneira, a presença de estruturas que inibiriam a ocupação por lianas poderia ser um investimento pouco eficiente para a árvore, e a competição com as outras plantas da vizinhança poderia ser igualmente importante.

Provavelmente a limitação de luz na floresta é um fator mais importante que os caracteres das árvores para evitar lianas. Lianas são plantas heliófilas que necessitam de luz para se reproduzir, uma vez que a maioria floresce e frutifica apenas no dossel da floresta (Campbell \& Newbery 1993). Dessa maneira, sua abundância está intimamente relacionada ao regime de luz e à dinâmica de clareiras. Madeira et al. (2009) relataram que a abundância de lianas é maior em estádios intermediários de sucessão ecológica, uma vez que nessa etapa há tanto a luz quanto a disponibilidade de suporte. 
Apesar das árvores do dossel apresentarem maior probabilidade de crescimento e reprodução, elas estão mais propensas a serem ocupadas por lianas, que podem diminuir sua taxa de crescimento e fecundidade e o forófito poderia investir em estruturas que diminuiriam a probabilidade de ocupação por lianas, como a presença de casca esfoliante (Hegarty 1991). Entretanto não encontramos relação entre a presença dessas estruturas nas árvores e a menor probabilidade de ocupação por lianas. Provavelmente a competição com outras árvores na floresta é forte o suficiente para que o investimento em caracteres que inibiram lianas seja irrelevante para o potencial forófito.

\section{Agradecimentos}

Os autores agradecem à Fundação de Amparo à Pesquisa do Estado de Minas Gerais e a Vale S.A., o financiamento do projeto RDP-00104-10, e ao CNPq, o financiamento do projeto 472250/2010-8. Agradecemos também à Marcela Alcantara, Luis Felipe Oliveira e Tatiana Carvalho, a ajuda na coleta dos dados em campo.

\section{Referências}

Balfour, D.A. \& Bond, W.J. 1993. Factors limiting climber distribution and abundance in a Southern African Forest. Journal of Ecology 81: 93-100.

Campanello, P.; Garibaldi, J.; Gatti, M. \& Goldstein, G. 2007. Lianas in a subtropical Atlantic Forest: host preference and tree growth. Forest Ecology and Management 242: 250-259.

Campbell, E.J.F. \& Newbery, D.M. 1993. Ecological relationships between lianas and trees in lowland Rain Forest in Sbah, East Malaysia. Journal of Tropical Ecology 9: 469-490.

Carsten, L.D.; Juola, F.A.; Male, T.D. \& Cherry, S. 2002. Host associations of lianas in a south-east Queensland rain forest. Journal of Tropical Ecology 18: 107-120.

Clark, D. B. \& Clark, D. A. 1990. Distribution and effects on tree growth of lianas and woody hemiepiphytes in a Costa Rican tropical wet forest. Journal of Tropical Ecology 6: 321-331.

Clark, D.A. \& Clark, D.B. 1992. Life history diversity of canopy and emergent trees in a neotropical rain forest. Ecological Monographs 62: 315-344.

Chen, Y.-J.; Bongers, F.; Cao, K.-F. \& Cai, Z.-quan. 2008. Above- and below-ground competition in high and low irradiance: tree seedling responses to a competing liana Byttneria grandifolia. Journal of Tropical Ecology 24: 517-524.

Chittibabu, C.V. \& Parthasarathy, N. 2001. Liana diversity and host relationships in a tropical evergreen forest in the Indian Eastern Ghats. Ecological Research 16: 519-529.

Costa, C.M.R. 1998. Biodiversidade em Minas Gerais: um atlas para a sua conservação. Fundação Biodiversitas, Belo Horizonte. 92p.

Dawkins, H.C. \& Field, D.R.B. 1978. A long-term surveillance system for British woodland vegetation. Oxford Forestry Institute, Oxford. 106p.

Ding, Y. \& Zang, R. 2009. Effects of logging on the diversity of lianas in a lowland tropical rain forest in Hainan Island, South China. Biotropica 41: 618-624.

Fonseca, M.G.; Vidal, E. \& Santos, F.A.M. 2009. Intraspecific variation in the fruiting of an amazonian timber tree: implications for management. Biotropica 41: 179-185.

Ghazoul, J. \& Sheil, D. 2010. Tropical rain forest - ecology, diversity, and conservation. Oxford University Press, Oxford. 516p.

Gerwing, J.J.; Schnitzer, S.A.; Burnham, R.J.; Bongers, F.; Chave, J.; DeWalt, S.J.; Ewango, C.E.N. et al. 2006. A standard protocol for liana censuses. Biotropica 38: 256-261.

Grogan, J. \& Landis, R.M. 2009. Growth history and crown vine coverage are principal factors influencing growth and mortality rates of big-leaf mahogany Swietenia macrophylla in Brazil. Journal of Applied Ecology 46: 1283-1291.

Hegarty, E.E. 1991. Vine-host interaction. In: Putz, F. E. \& Mooney H.A. (eds.). The biology of vines. Cambridge University Press, Cambridge. Pp. 357-376.

Homeier, J.; Englert, F.; Leuschner, C.; Weigelt, P. \& Unger, M. 2010. Factors controlling the abundance of lianas along an altitudinal transect of tropical forests in Ecuador. Forest Ecology and Management 259: 1399-1405.

Jiménez-Castillo, M. \& Lusk, C.H. 2009. Host infestation patterns of the massive liana Hydrangea serratifolia (Hydrangeaceae) in a Chilean temperate rainforest. Austral Ecology 34: 829-834.

Kainer, K.A.; Wadt, L.H. O.; Gomes-Silva, D.A.P. \& Capanu, M. 2006. Liana loads and their association with Bertholletia excelsa fruit and nut production, diameter growth and crown attributes. Journal of Tropical Ecology 22: 147-154.

Legendre, P. \& Fortin, M.-J. 1989. Spatial pattern and ecological analysis. Vegetatio 80: 107-138.

Madeira, B.G.; Espírito-Santo, M.M.; Neto, S.D.; Nunes, Y.R.F.; Azofeifa, G.A.S., Fernandes, G.W. \& Quesada, M. 2009. Changes in tree and liana communities along a successional gradient in a tropical dry forest in south-eastern Brazil. Plant Ecology 201: 291-304.

Malizia, A. \& Grau, H.R. 2006. Liana - host tree associations in a subtropical montane forest of north-western Argentina. Journal of Tropical Ecology 22: 331-339. 
McLeod, A.I. 2011. Kendall: Kendall rank correlation and Mann-Kendall trend test. R package version 2.2. Disponível em <http://CRAN.R-project.org/ package $=$ Kendall $>$. Acesso em 7 Jul 2012.

Muthuramkumar, S. \& Parthasarathy, N. 2001. Treeliana relationships in a tropical evergreen forest at Varagalaiar, Anamalais, Western Ghats, India. Journal of Tropical Ecology 17: 395-409.

Pérez-Salicrup, D.R.; Sork, V.L. \& Putz, F.E. 2001. Lianas and trees in a liana forest of Amazonian Bolivia. Biotropica 33: 34-47.

Pinard, M.A. \& Putz, F.E. 1994. Vine infestation of large remnant trees in logged forest in Sabah, Malaysia: biomechanical facilitation in vine succession. Journal of Tropical Forest Science 6: $302-309$.

R Development Core Team. 2011. R: A language and environment for statistical computing. $\mathrm{R}$ Foundation for Statistical Computing, Vienna, Austria. Disponível em <http://www.R-project. org/>. Acesso em 7 Jul 2012.
Reddy, M.S. \& Parthasarathy, N. 2006. Liana diversity and distribution on host trees in four inland tropical dry evergreen forests of peninsular India. Tropical Ecology 47: 109-123.

Schnitzer, S.A.; Kuzee, M.E. \& Bongers, F. 2005. Disentangling above- and below-ground competition between lianas and trees in a tropical forest. Journal of Ecology 93: 1115-1125.

Schnitzer, S.A.; Rutishauser, S. \& Aguilar, S. 2008. Supplemental protocol for liana censuses. Forest Ecology and Management 255: 1044-1049.

Talley, S.M.; Setzer, W.N. \& Jackes, B.R. 1996. Host associations of two adventitious-root-climbing vines in a north Queensland tropical rain forest. Biotropica 28: 356-366.

Torres, R.B.; Kinoshita, L.S. \& Martins, F.R. 1994. Aplicação de padrões de casca na identificação de árvores da Estação Ecológica de Angatuba, SP. Revista Brasileira de Botânica 17: 119-127.

Zar, J.H. 2010. Biostatistical analysis. $5^{\text {th }}$ ed. Prentice Hall, New Jersey. 944p. 